but damage to the tissues by pressure and, nearly always, cause enlargement of the cup. The rarefaction could only exist for the pathologist to find if the pressure were not raised. But from the similarity in the cups and visual effects to glaucoma, may not Schnabel have confused the low tension group with the glaucoma group, and really have described the former?

Although " pseudoglaucoma" has been used for certain congenital conditions of the disc cup, yet it would appear to be for the time being a good name to appropriate to this group of enlarged cups in adults without a rise in the intra-ocular tension; pending an investigation in its pathology, which would probably provide a better. This name suggests its similarities to glaucoma, but distinguishes it from that group of symptoms.

In conclusion I wish to thank Prof. Newman for his help and advice in the compilation of this paper.

\title{
REFERENCES
}

(a) Pickard, R.-Red field and optic disc resistance in glaucoma and allied conditions. Proc. R. Soc. of Med., Vol. XXIII, Sect. of Ophthal., pp. 57-76, 1930.

(b) Cavernous optic atrophy and its relation to glaucoma. Brit. Jl. of Ophthal., Vol. IX, p. 385, 1925.

(c) The visual field in atheroma of the retinal vessels. Proc. $R$. Soc. of Med., Vol. XIX, Sect. of Ophthal., pp. 55-75, 1926.

(d) Cridland, B.-The tonometer of Schiötz. Brit. Jl. of Ophthal., Vol. I, p. 354, 1917.

\section{A NEW PORTABLE CORNEAL MICROSCOPE}

BY

\section{J. LONGWORTh Blair}

LON DON

IN the diagnosis of certain ophthalmological conditions the necessity for instruments giving high magnification and appropriate lighting has been demonstrated by the popularity of the binocular corneal microscope used in conjunction with some form of slit-lamp. This involves large apparatus which must be fixed. The instrument here described is designed to give a reasonable amount of magnification combined with sufficient illumination in a portable form. The instrument for the purpose of description may be divided into :-

$a$. The microscope and stand. $b$. The source of illumination. The microscope consists of a small optical unit capable of magnification of $\times 10$ to $\times 20$ inclusive, mounted on a stand for application 
to the patient's forehead and with screw adjustment. The stand itself is capable of being adjusted to meet the discrepancies in slope of various foreheads.

The source of illumination consists of a lamp and its mounting. The lamp is a small luminous ophthalmoscope bulb with a single filament in a small cylindrical case with a condensing lens which

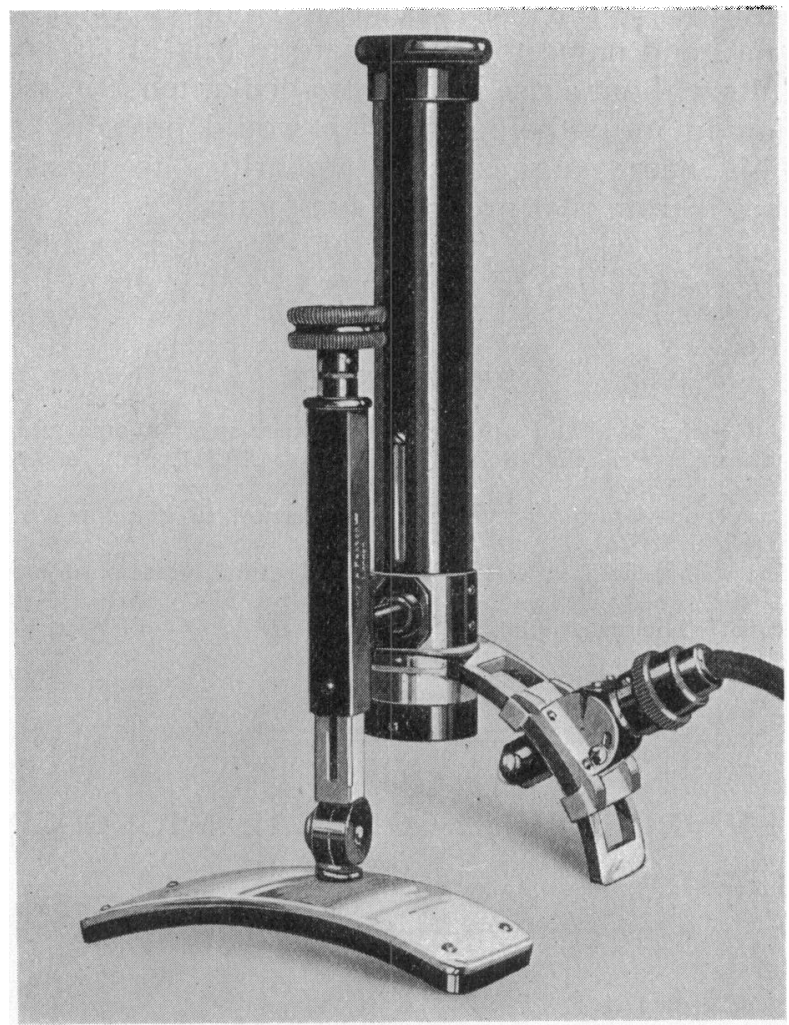

is capable of adjustment. There are two stops provided, one of which gives a sharp line, being the focus of the filament, and the other gives an even disc. The sharp line is the more important and is called "the focus of the lamp" in the remainder of this description.

The lamp is mounted on a slide travelling upon a quadrant of such a design as to combine ease of movement along the quadrant with rigidity in other directions.

The quadrant is mounted on the objective end of the microscope in such a way that it may be swung through $180^{\circ}$ about the axis of the microscope. 
The lamp itself has two movements, one a rotation about its own axis. By this means the line of light called "the focus of the lamp " may be adjusted to be horizontal or vertical to the line of incidence. The other movement is a rotation in the plane of the quadrant, and by this means the angle of incidence of the light may be varied. There is a mark which indicates the position of the lamp, which will bring the "focus of the lamp " and the focus of the microscope, when adjusted for a magnification of 10 , on to the same point.

The current needed to light the bulb is obtained from a small dry battery which can be carried in the pocket.

\section{As to use}

Assuming the right eye is to be examined. The most convenient position for the patient is lying on a couch in a horizontal plane, the observer standing behind the head.

The stand is then placed on the patient's forehead with the light sharply focussed on the object to be viewed. Coarse adjustments can be made by moving the stand or flexing the joint. As the focus of the microscope coincides with that of the light, the object is brought immediately into view. The whole instrument is then steadied by the observer with the palm of the left hand leaving the fingers free to control the lids of the patient. Finer adjustments may then be made with the right hand by means of the screw.

The right hand is then left free, either further to steady the instrument or for such manipulation as removing a foreign body, etc. Variations in the obliquity of the beam of light may also be made by sliding the lamp on the quadrant.

For the left eye swing the quadrant through $180^{\circ}$ and hold stand with right hand leaving left free.

This instrument is suggested for use as an aid to diagnosis where the ordinary loupe is not sufficient and a slit-lamp is not available, to determine depth of foreign bodies in a cornea and to remove minute foreign bodies where skilled assistance in holding and focussing a lamp is not available.

It is also hoped that this instrument will assist surgeons who are unable to use a binocular microscope or loupe through lack of balance in their extra-ocular muscles. 